\title{
Comparison of the in vitro invasive capabilities of Plasmodium falciparum schizonts isolated by Percoll gradient or using magnetic based separation
}

\author{
Carmenza Spadafora ${ }^{1,2^{*}}$, Lucia Gerena ${ }^{3}$ and Karen M Kopydlowski,
}

\begin{abstract}
Background: Percoll gradient centrifugation is often used for synchronization, enrichment, or isolation of a particular stage of Plasmodium falciparum. However, Percoll, a hyperosmotic agent, may have harmful effects on the parasites. Magnetic bead column (MBC) separation has been used as an alternative. This is a report of a headto-head comparison of the in vitro invasive capabilities of parasites isolated by either of the two methods.

Methods: The P. falciparum laboratory strain isolate $7 \mathrm{G} 8$ was grown in vitro using standard procedures and synchronized using 5\% sorbitol. On separate days when the schizont parasitaemia was $>1 \%$, the culture was split and half was processed by Percoll gradient centrifugation and the other half by magnetic bead column separation. Both processed parasites were placed back in culture and allowed to invade new uninfected erythrocytes.

Results: In 10 paired assays, the mean efficiency of invasion of 7G8 parasites treated by Percoll gradient centrifugation was $35.8 \%$ that of those treated by magnetic bead column separation $(95 \% \mathrm{Cl}, \mathrm{p}=0.00067)$ A paired $t$ test with two tails was used for these comparisons.

Conclusions: In this comparison, magnetic bead column separation of $7 G 8$ schizonts resulted in higher viability and efficiency of invasion than utilizing Percoll gradient centrifugation.
\end{abstract}

\section{Background}

Purification of schizonts from in vitro cultures of Plasmodium falciparum is desirable for a number of reasons: to synchronize the culture and aid in the study of the stage specific proteome and transcriptome, to carry out red cell invasion assays in order to study mechanisms of merozoite invasion, to test the growth inhibitory activity of antibodies or anti-sera, and to determine the stage specific efficacy of potential anti-malarial drugs. To achieve synchronization several methods have been developed. One of the earliest reported is based on the use of a gelatin [1], which relies on the different sedimentation speeds of the infected erythrocytes dependent on the age of the parasite inside. It was reported that a better substitute (i.e., Plasmion) of the common, but discontinued Plasmagel, works equally well regardless of the strain used [2], since only membrane

\footnotetext{
* Correspondence: cspadafora@indicasat.org.pa

${ }^{1}$ Cellular Injury Department, Division of Military Casualty Research, Walter Reed Army Institute of Research, 503 Robert Grant Ave., Silver Spring, MD 20902, USA

Full list of author information is available at the end of the article
}

knobs expressing strains could be separated with Plasmagel [3]. The most common method used by researchers to synchronize their cultures is incubation with $5 \%$ sorbitol [4], which destroys the late stages of the parasite (i.e., throphozoites and schizonts). This method is very toxic to the parasites, though, and the researcher usually has to allow for the parasites to complete up to two more reproduction cycles for full recovery from the treatment [5-7]. Another way of separating the stages is through the use of a discontinuous gradient of Percoll [8] with or without sorbitol, or in combination with glucose. Percoll gradient centrifugation became the method of choice by most malaria researchers when trying to separate different stages of the parasitized erythrocytes. A method based on the magnetic properties of the late trophozoite or schizont stage of the malaria parasite was later described by Staalsoe [9], and the viability of the parasites treated this way was tested in vivo by Thi Trang [10]. The use of magnetic bead columns to capture malaria infected erythrocytes is possible because Plasmodium parasites utilize the haemoglobin of the red blood cells as a nutrient and transform the iron 
containing haem group into haemozoin, a pigment that remains in the food vacuole. Therefore, schizonts possess an abundance of the iron-containing haemozoin. Because the iron in haemozoin is in the $\mathrm{Fe}^{3+}$ state, it has a stronger paramagnetic effect than the iron in haemoglobin, which is in the $\mathrm{Fe}^{2+}$ state. This translates into a stronger magnetic attraction under a magnetic field for haemozoin than for haemoglobin. Because not all strains of P. falciparum seem to withstand well the Percoll treatment [11], the in vitro retained capability of invasion of new uninfected erythrocytes of the 7G8 strain parasites after purification either by Percoll gradient centrifugation or magnetic bead column separation was compared.

\section{Methods}

\section{Parasites and culture}

Plasmodium falciparum chloroquine-resistant and pyrimethamine-resistant strain 7G8 [12] was used throughout. The parasites were grown as per the method described by Haynes et al [13] using O+ red blood cells at 2-4\% haematocrit in RPMI 1640 containing $25 \mathrm{mM}$ Hepes, 2\% glucose, $0.2 \%$ sodium bicarbonate, (culture media, CM) plus $10 \%$ $\mathrm{O}+$ plasma or serum. Semi-synchronized cultures with 1-10\% parasitaemia were used to isolate RBCs infected with late stages of the parasite. Giemsa-stained smears of the culture and flow cytometry by Hoechst 33342 staining of the purified parasites were prepared as needed to evaluate parasitaemia.

\section{Percoll gradient and magnetic column separation}

Cultures with parasitaemia of 4-20\% were divided into two equal portions. One half was used for Percoll gradient centrifugation and the other for magnetic bead column separation. The Percoll gradient consisted of three layers of Percoll in RPMI (from top to bottom: $40 \%$ $(4 \mathrm{ml}), 70 \%(6 \mathrm{ml})$ and $90 \%(8 \mathrm{ml})$ Percoll $)$ placed in 30 ml OAK RIDGE polycarbonate screw cap centrifuge tubes. The culture was centrifuged at $3000 \mathrm{rpm}$ for $5 \mathrm{~min}$ at $4^{\circ} \mathrm{C}$, the pellet resuspended in RPMI-1640 $(1: 1 \mathrm{vol} / \mathrm{vol})$, and carefully placed on top of the gradient. The tubes were centrifuged at $10,000 \mathrm{rpm}$ for $20 \mathrm{~min}$ at $20^{\circ} \mathrm{C}$. The cells at the $40-70 \%$ interface, containing mostly schizonts, were collected, washed twice in RPMI1640 , resuspended in culture medium, counted in a haemocytometer and used as inoculum for the overnight cultures. For magnetic column separation, we used MACS Separation Columns "LS" (Miltenyi Biotec, Germany). Pre-equilibration, washings, and elution were all carried out at RT with $\mathrm{CM}$. Upon applying a magnetic field, three $8 \mathrm{ml}$ loads of culture were added to the column and the flow-through, which usually contained uninfected erythrocytes, ring-infected erythrocytes, and young trophozoites, was collected and used to reload the column or was placed back in culture. After one wash with $8 \mathrm{ml}$ of $\mathrm{CM}$ (since then we have optimized the purity obtained with the procedure by washing first with one $\mathrm{ml}$ followed by $5 \mathrm{ml}$ of $\mathrm{CM}$ ), the column was removed from the magnetic field and its contents eluted with $3 \mathrm{ml}$ of CM. The eluted parasitized RBCs were centrifuged at 1,500 rpm for $5 \mathrm{~min}$ at RT, the supernatant discarded, and the pellet resuspended in 150-250 $\mu \mathrm{l}$ of $\mathrm{CM}$ plus $10 \%$ human serum (CMS), depending on the concentration desired. The number of parasites captured was calculated by haemocytometer determination, as with the Percoll treatment.

\section{Invasion assays}

Schizont-infected erythrocytes from both magnetic beads column or Percoll gradient separation were incubated for 16-22 hrs at a haematocrit of 1-2\% in CMS, in duplicate wells. Final schizont-infected parasitaemia ranged from 1 to 10\%. For light microscopy readings, Giemsa-stained thin smears were prepared from each well and the number of ring-infected erythrocytes in 1,000 erythrocytes was counted. The microscopist was always blinded to the experimental group designation of each slide. For the flow cytometry readings, infected red blood cells were stained with Hoechst 33342 and read with a UV laser in a BD LSRII equipment following procedures as described by Spadafora et al [14]. Each experiment was set up in duplicate or triplicate wells, and invasion rates were averaged between all the wells.

\section{Statistical analysis}

The percentage of efficiency of invasion compared to the MCB method is the result of the following formula:

$$
\frac{\text { Average invasion Percoll }}{\text { Average invasion MBC }} \times 100=\% \text { Efficiency of invasion }
$$

A paired t-test analysis was performed, where each sample from Percoll and from MBC done on the same day was compared to each other for their invasion percent into new red blood cells. Only experiments with purity of isolation above $65 \%$ were considered for the calculations.

\section{Results and Discussion}

Table 1 shows the results of the experiments. In 10 paired assays, the mean percent invasion of intact red cells after overnight culture was $5.3 \%$ for magnetic bead column separation and $1.9 \%$ for Percoll gradient centrifugation, accounting for a $39.5 \%$ efficiency of invasion by the parasites treated with the Percoll method compared to the magnetic beads one $(95 \% \mathrm{CI}, \mathrm{P}=0.00067)$.

It is evident that schizonts purified by magnetic bead column separation were more viable than those purified by Percoll gradient centrifugation. The reasons for this 
Table 1 Relationship between $P$. falciparum capacity of invasion after two different separation treatments

\begin{tabular}{ccccc}
\hline Experiment & $\begin{array}{c}\text { Initial } \\
\text { Parasitaemia }\end{array}$ & Replicates & $\begin{array}{c}\text { Percoll } \\
\text { Invasion }\end{array}$ & $\begin{array}{c}\text { MB } \\
\text { invasion }\end{array}$ \\
\hline${ }^{* 1}$ & $10 \%$ & 2 & $0.8 \%$ & $8.2 \%$ \\
${ }^{*} 2$ & $4 \%$ & 2 & $0.6 \%$ & $2.0 \%$ \\
${ }^{*} 3$ & $2 \%$ & 3 & $1.3 \%$ & $2.4 \%$ \\
${ }^{*} 4$ & $2 \%$ & 3 & $1.9 \%$ & $3.8 \%$ \\
5 & $2 \%$ & 2 & $2.0 \%$ & $6.7 \%$ \\
6 & $5 \%$ & 2 & $4.9 \%$ & $7.3 \%$ \\
7 & $1 \%$ & 2 & $0.8 \%$ & $4.4 \%$ \\
8 & $2 \%$ & 3 & $2.8 \%$ & $11.5 \%$ \\
9 & $2 \%$ & 3 & $2.0 \%$ & $4.1 \%$ \\
10 & $2 \%$ & 3 & $1.7 \%$ & $2.7 \%$ \\
Average: & & & $1.9 \%$ & $5.3 \%$ \\
\hline Parites & & & &
\end{tabular}

Parasites underwent separation by use of a Percoll gradient (Percoll invasion column) or passage through a magnetic beads column (MB invasion column). The collected schizonts were then placed in culture with new red blood cells to allow for a new round of infection by the parasite. The parasitaemia was calculated by flow cytometry except for experiments marked with " "**" which were determined by light microscopy.

are not clear. However, there are several possible explanations. It hs been reported that permeabilization of the host erythrocyte membrane appears s early as $6 \mathrm{~h}$ after parasite invasion and increases gradually with parasite maturation [15]. Since the collection of parasites for next day invasion need collection of schizont forms, the erythrocyte is at its peak of permeability. This suggests that molecules could diffuse in and out of the schizontinfected erythrocyte with relative ease, perhaps leading to increased susceptibility to toxic substances that could be present in Percoll. Another possible explanation for the decreased viability after Percoll centrifugation is the leakage of ions across the membrane due to the hyper osmotic effect of Percoll.

Bates et al [16] reported that a group of laboratory established strains of $P$. falciparum treated by two methods of isolation, MCB and a centrifugation gradient obtained with Percoll to which sorbitol has been added, did not show any difference in their invasion capabilities. However, they reported that when they performed the same analysis with field isolates their results were' similar to those we show here, with the MCB treated ones invading at higher rates. It seemed to them that the adaptation to the laboratory conditions was the major factor determining if the parasites would be affected or not by the Percoll/sorbitol treatment. In view of the present experiments, these results are better explained by the difference in susceptibility to external chemicals and solvents inherent to different strains. Reed et al [11] reported that the laboratory-adapted strain W2mef could not be isolated using the Percoll method, which would also point out to different strains behaving differently to the Percoll treatment, for reasons that are still unknown, and that could include different membrane porosity and osmotic pressure sensitivity. In the case of 7G8, the isolation is possible but the invasion efficiency is reduced, something that could be avoided using the magnetic beads based technique.

In addition to increased viability, magnetic bead column separation offers the advantage that the parasitized red cells remain in an iso-osmotic environment. This means that the red cells can be introduced back into the culture media without concern for abrupt changes in the osmolarity, which could affect some strains more than others.

\section{Conclusions}

Magnetic column enrichment of schizont and late stage trophozoite-infected red cells is a very viable alternative to Percoll gradient centrifugation especially for the purpose of preparing red cell invasion assays with the 7G8 strain. This technique can be recommended for several reasons: the procedure itself has the advantage of not being invasive on the parasites since it involves no contact with any foreign chemical reagent to the culture media, it requires no expensive centrifuges, and 7G8, as well as some other strains seem to be affected by the use of Percoll gradient centrifugation.

\section{Acknowledgements}

The authors would like to thank Christian Darko (Naval Medical Research Center) for introducing us to the use of magnetic columns. Sheila Peel (The Walter Reed Army Institute of Research, HIV Program) gave very helpful advice on the culture of the parasites and analysis of the data. KMK and LG were supported by the USAMRMC (U.S. Army Medical Research and Materiel Command) Military Infectious Diseases Research Program. CS was supported in part by a training grant from the Fogarty International Center (1 D43 TW06239, PI Jose A. Stoute), by intramural funds from the Uniformed Services University of Health Sciences, Bethesda, MD, and a training grant from the Secretaría Nacional de Ciencia y Tecnología (SENACYT), República de Panamá. The opinions expressed herein are those of the authors and do not reflect the views or opinions of the U.S. Army and the Department of Defense.

\section{Author details}

'Cellular Injury Department, Division of Military Casualty Research, Walter Reed Army Institute of Research, 503 Robert Grant Ave., Silver Spring, MD 20902, USA. ${ }^{2}$ Centro de Biologia Celular y Molecular de las Enfermedades, Instituto de Investigaciones Científicas y Servicios de Alta Tecnología-AIP (INDICASAT-AIP), Ciudad del Saber, Clayton, Panamá. ${ }^{3}$ Parasitology Department, Division of Experimental Therapeutics, Walter Reed Army Institute of Research, 503 Robert Grant Ave., Silver Spring, MD 20902, USA. ${ }^{4}$ US Army Medical Materiel Development Activity (USAMMDA), Fort Detrick, MD 21702, USA.

\section{Authors' contributions}

CS suggested the idea, developed the protocol for the magnetic beads separation, maintained in vitro culture, carried out most parts of the laboratory experiments, prepared and analyzed the data obtained, drafted the paper, and finalized the manuscript for submission with contribution from authors. KMK was involved in the experimental design, participated in the coordination of the project under which this study was conducted, assisted in the management of culture, helped supervise, and contributed to the preparation of the manuscript. LG contributed with maintaining parasite culture and assisted with interpretation of smears. All authors read and approved the final manuscript. 


\section{Competing interests}

The authors declare that they have no competing interests.

Received: 22 February 2011 Accepted: 18 April 2011

Published: 18 April 2011

\section{References}

1. Jensen JB: Concentration from continuous culture of erythrocytes infected with trophozoites and schizonts of Plasmodium falciparum. Am J Trop Med Hyg 1978, 27:1274-1276.

2. Lelièvre J, Berry A, Benoit-Vical F: An alternative method for Plasmodium culture synchronization. Exp Parasitol 2005, 109:195-197.

3. Pasvol G, Wilson RJ, Smalley ME, Brown J: Separation of viable schizontinfected red cells of Plasmodium falciparum from human blood. Ann Trop Med Parasitol 1978, 72:87-88.

4. Lambros C, Vanderberg JP: Synchronization of Plasmodium falciparum erythrocytic stages in culture. J Parasitol 1979, 65:418-420.

5. Radfar A, Mendez D, Moneriz C, Linares M, Marin-Garcia P, Puyet A, Diez A, Bautista JM: Synchronous culture of Plasmodium falciparum at high parasitemia levels. Nat Protoc 2009, 4:1899-915.

6. Krugliac M, Zhang J, Nissani E, Steiner-Mordoch S, Ginsburg H: Killing of intraerythrocytic Plasmodium falciparum by lisosomotropic amino acid esters. Parasitol Res 2003, 89:451-458.

7. Butcher GA, Clark IA: Plasmodium falciparum growth in vitro by sera from mice infected with malaria or treated with TNF. Parasitology 1990, 101:321-326.

8. Nillni EA, Londner MV, Spira DT: A simple method for separation of uninfected erythrocytes from those infected with Plasmodium berghei and for isolation of artificially released parasites. Z Parasitenkd 1981, 64:279-284.

9. Staalsoe T, Giha HA, Dodoo D, Theander TG, Hviid L: Detection of antibodies to variant antigens on Plasmodium falciparum-infected erythrocytes by flow cytometry. Cytometry 1999, 35:329-336.

10. Trang DT, Huy NT, Kariu T, Tajima K, Kamei K: One-step concentration of malarial parasite-infected red blood cells and removal of contaminating white blood cells. Malar J 2004, 3:7.

11. Reed MB, Caruana SR, Batchelor AH, Thompson JK, Crabb BS, Cowman AF: Targeted disruption of an erythrocyte binding antigen in Plasmodium falciparum is associated with a switch toward a sialic acid-independent pathway of invasion. Proc Natl Acad Sci USA 2000, 97:7509-7514.

12. Burkot TR, Williams $J$, Schneider I: Infectivity to mosquitoes of Plasmodium falciparum clones grown in vitro from the same isolate. Trans R Soc Trop Med Hyg 1984, 78:339-341.

13. Haynes JD, Diggs $C L$, Hines FA, Desjardins RE: Culture of human malaria parasites Plasmodium falciparum. Nature 1976, 263:767-769.

14. Spadafora C, Awandare GA, Kopydlowski KM, Czege J, Moch JK, Finberg RW, Tsokos GC, Stoute JA: Complement receptor 1 is a sialic acidindependent erythrocyte receptor of Plasmodium falciparum. PLOS Pathog 2010, 6:e1000968.

15. Kutner S, Breuer W, Ginsburg H, Aley SB, Cabantchik Zl: Characterization of permeation pathways in the plasma membrane of human erythrocytes infected with early stages of Plasmodium falciparum: association with parasite development. J Cell Physiol 1985, 125:521-527.

16. Bates $A H, M u J$, Jiang $H$, Fairhurst RM, Su X: Use of magnetically purified Plasmodium falciparum parasites improves the accuracy of erythrocyte invasion assays. Exp Parasitol 2010, 126:278-280.

doi:10.1186/1475-2875-10-96

Cite this article as: Spadafora et al.: Comparison of the in vitro invasive capabilities of Plasmodium falciparum schizonts isolated by Percoll gradient or using magnetic based separation. Malaria Journal 2011 10:96.

\section{Submit your next manuscript to BioMed Central and take full advantage of:}

- Convenient online submission

- Thorough peer review

- No space constraints or color figure charges

- Immediate publication on acceptance

- Inclusion in PubMed, CAS, Scopus and Google Scholar

- Research which is freely available for redistribution

Submit your manuscript at www.biomedcentral.com/submit
Biomed Central 